\title{
REVIEW ARTICLE Spatial light-scattering analysis as a means of characterizing and classifying non-spherical particles
}

\author{
Paul H Kaye
}

Engineering Research and Development Centre, University of Hertfordshire, Hatfield, Hertfordshire AL10 9AB, UK

Received 15 May 1997, accepted for publication 4 August 1997

\begin{abstract}
The manner in which a particle scatters incident light is a function of the particle's shape, its size, its structure and its orientation with respect to the illumination direction, as well as to the wavelength and polarization state of the light. The spatial intensity distribution of scattered light thus contains information by which the particle may often be classified or even identified. This paper briefly reviews the development of spatial light scattering analysis as an analytical and monitoring tool now finding application in areas as diverse as environmental monitoring, aerosol and hydrosol research, microbiology and fine-powder characterization. The paper concludes with a practical example of the use of spatial scattering analysis to achieve real-time discrimination of a specific particle type, namely airborne asbestos fibres.
\end{abstract}

\section{Introduction}

In a wide variety of environmental, occupational and industrial scenarios, fine particles, typically within the size range from a few tenths of a micrometre to a few hundred micrometres, play an important role. Airborne particles can contaminate industrial processes and can present a respirable health hazard, such as in the case of asbestos and other mineral fibres. Liquid-borne particles may be similarly undesirable, for example in the cases of bacterial contamination of processed drinking water and of contamination by solid particles or immiscible droplets of petroleum-based fuels, or hydraulic fluids. Moreover, in an increasing number of industrial processes involving pharmaceuticals, foodstuffs, pigments, ceramics, and so on, products may pass through a fine powder phase during production or are rendered into a fine powder form for eventual sale.

The majority of these particles (with the exception of airborne droplets and immiscible liquid-liquid droplets) will to some extent be non-spherical. In many cases a knowledge of the shape of particles may therefore be used to advantage. For example, in environmental and occupational scenarios, the shape of particles (such as fibrous, flake-like, deformable microdroplet, irregular cubic) can give some indication of the source of those particles and hence facilitate more effective contamination control and measures designed to reduce inadvertent particle generation. Additionally, certain particle types may be identified against a background of other particulates by virtue of their shape: respirable airborne fibres are an important example of this type. In industrial product processing, particle shape and size distributions can affect the quality or efficacy of the finished product through factors such as packing efficiencies, texture and mechanical strength.

Although optical and electron microscopies together with manual or automated image analysis can provide substantial information relating to the particle shape and size, these techniques are generally laborious and are unsuited to monitoring and process-control applications in which a rapid response to shape information is normally required. Where such measurements are desirable, optical scattering methods have become widely accepted. These may be broadly classified into ensemble-scattering and single-particle-scattering techniques. In general, the ensemble-scattering technique, whereby the scattered light is recorded simultaneously from a multitude of randomly orientated particles, is capable of providing particle-sizedistribution information but is unable to provide comparable data relating to particle shapes because of averaging effects. Exceptions to this have been demonstrated by Rarity (1987), who measured the asymmetry of ellipsoid-like particles in suspension by investigating intensity fluctuations using cross correlation techniques, and more recently by Heffels et al (1994), who determined particle-shape information from low-concentration particle suspensions by using a similar analysis of the temporal and azimuthal fluctuations in the forwards-scattered light.

The alternative technique, based on single-particle scattering, is the basis of numerous commercial particle-counting-sizing 
instruments. In general, these instruments use a single detector to collect light scattered by individual particles carried in a fluid flow. The magnitude of the detector's output is related to the size of the scattering particle via calibration tables which are usually based upon spherical particle scattering. These instruments are not designed to assess particle shapes and indeed may yield erroneous sizing data when confronted with non-spherical particles. Considerable effort has therefore been devoted to characterizing their response to non-spherical particles (see for example Bottlinger and Umhauer (1987), Gebhart and Anselm (1987), Killinger and Zerull (1987) and Umhauer and Bottlinger (1991)).

It is only when multiple detectors are employed to examine the spatial distribution of the light scattered by individual particles that information relating to the particle shape as well as size may be determined. Hence this paper reviews the development of the field of single-particle spatial light scattering and concludes with an example of how the method has been employed in a practical example to solve a specific particle-characterization and discrimination problem.

(Note that a hybrid approach has been described by Neeße et al (1995), who successfully adapted a light-scattering instrument usually employed for ensemble particle-sizing studies to obtain shape information relating to individual micro-cuboids. The method relied on having a single particle within the scattering cell and observing the temporal behaviour of the light diffracted by the particle as it was rotated by a turbulent flow field.)

\section{Spatial light scattering}

Although several workers, for example, Rochon et al (1988), Al-Chalabi et al (1990) and Spinrad and Brown (1993), have successfully employed measurement of the polarization state of the scattered light at a single scattering angle to yield particle-shape information (the first to assess the fraction of spherical particles contained within a particle population, the second to discriminate among and size, airborne fibres and the third to measure the asphericities of hydrosols), it is the spatial variation of the intensity of the scattered light which has attracted the most attention. It is that which is considered here.

In theory, the detailed spatial intensity distribution of light scattered by an individual particle (the spatial scattering profile) is a complex function of the particle's size, shape and orientation with respect to the incident illumination, as well as of the wavelength and polarization state of the incident illumination. It is normal to refer to the particle's size in relation to the wavelength $\lambda$ of the illumination by the dimensionless size parameter $\alpha$, where $\alpha=2 \pi \mathrm{a} / \lambda$, a being the effective radius of the particle (which is assumed to be spherical). If the particle is very much smaller than the wavelength of the illuminating radiation $(\alpha>1)$ and its relative refractive index is close to unity, the particle behaves as an oscillating dipole, radiating in all directions. In this, the so-called Rayleigh-scattering region (Bohren and Huffman 1983), the amount of light scattered at a specific angle is primarily a function of the volume of the particle and is thus insensitive to the particle shape (Gebhart 1976). This therefore sets a lower limit on the sizes of particles whose shapes can be investigated using visible radiation and, in practical terms, this limit corresponds to particle sizes of a few tenths of a micrometre for visible illumination. As the particle size increases, $(\alpha>1)$, the spatial scattering or scattering profile becomes increasingly complex, until, for particles very much larger than the wavelength of the illuminating radiation, concepts from geometrical optics, such as reflection and refraction, can be used to describe the spatial scattering and infer the particle shape. For practical reasons, such as delivery of particles singly into the illumination measurement space, the upper limit of the particle size in single-particle spatial scattering systems is usually of the order of a few hundred micrometres.

The spatial scattering by a single particle may be described in terms both of the polar scattering $\theta$ (namely $0-180^{\circ}$ scattering in a plane containing the axis of the incident illumination) and of the azimuthal scattering $\varphi$ (namely $360^{\circ}$ around the illumination axis at a fixed polar angle). Investigators have studied these parameters both separately and in combination, as is discussed briefly in the following sections.

\subsection{Polar scattering in a single azimuthal plane}

The most commonly used approach to the measurement of the spatial light scattering from a particle has been to measure polar scattering in a single azimuthal plane, that is, to measure the intensity of light around the particle in a single plane containing the illuminating beam. Figure 1 is a typical polar plot of this type for the scattering from an individual spherical particle having a size parameter $\alpha=50$. The plot was computed using Mie theory (Bohren and Huffman 1983), which allows exact solutions to be determined for spherical homogeneous particles. Conversely, interpretation of the polar intensity variation from an unknown particle can be used as a means of analysing and characterizing the particle (the so-called inverse problem), which led Wyatt (1968), for example, to postulate that unicellular micro-organisms may ultimately be identified by the manner in which they scatter light. Indeed, this work resulted in commercial scattering instruments (Wyatt 1972) and a succession of refinements to the technology by various other workers, including, for example, Ludlow (1979), who used an array of optical fibres to record scattering throughout the polar arc of approximately 180 about scattering particles in liquid flow, and Bartholdi et al (1980), who were able to record almost the complete 360 circle of scattering about single particles in flow by means of an annular ellipsoidal reflector which surrounded the particle and directed scattered light onto a dedicated photodiode detector array.

The major limitation of this type of scattering geometry for single-particle characterization is that the measured intensity distributions are, as stated earlier, profoundly affected by the particle shape and therefore by the particle 


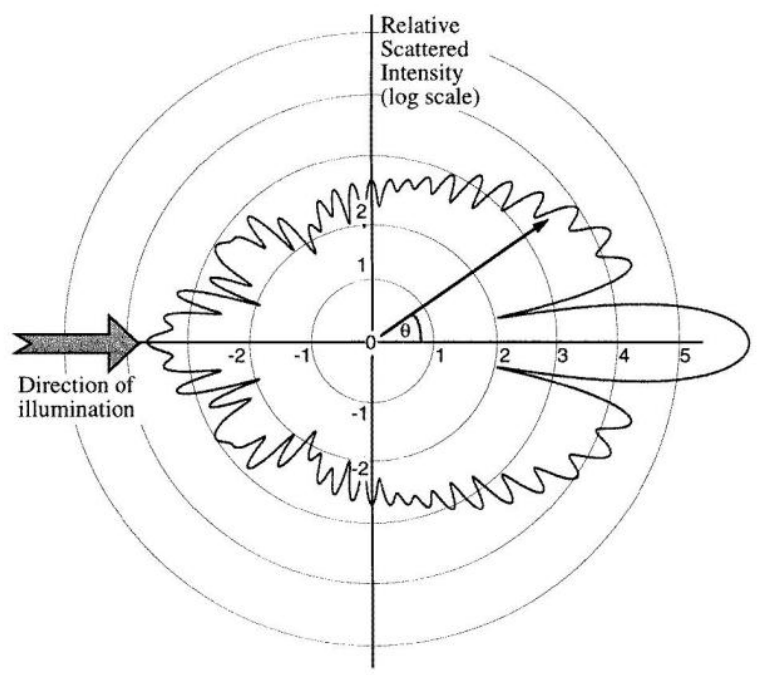

Figure 1. A theoretical polar plot showing the relative intensity of light scattered by a spherical particle with a size parameter $\alpha=\mathbf{5 0}$ and a refractive index of 1.33. Similar types of pattern from unknown particles (assumed spherical) can be interpreted to yield information on the particle size and refractive index (the inverse problem).

orientation (Latimer et al 1978, Zerull et al 1986). This has meant that most detailed studies of particle structure have been restricted to particles which exhibit spherical symmetry, such as was the case in a study of protoplast dehydration in bacterial spores by Ulanowski and Ludlow (1993). However, other workers have used this shape sensitivity to their advantage. For example, Allen and Husar (1976) used a system of diametrically opposed detectors, as shown in figure 2, to measure light scattered by airborne particles and were able to distinguish certain limited particle shapes by taking the ratio of the sums and differences of the two detector outputs; Diehl et al (1979) similarly used the ratio of detector outputs at various pairs of scattering angles, as illustrated in figure 3, to distinguish fibrous from non-fibrous particles in liquid suspension.

\subsection{Azimuthal scattering at a single polar angle}

The alternative to analysing the polar intensity variation within a single azimuthal plane about the scattering particle is to consider the azimuthal scattering, namely the radial intensity variation of light scattered at a constant polar scattering angle $\theta$ about the propagation axis of the illuminating light. A spherical homogeneous particle illuminated by circularly polarized light will result in a pattern of scattered light which is radially symmetrical about the axis of the illuminating beam. Conversely, non-spherical particles will result in intensity variations dependent both on the shape and on the orientation of the particle. An instrument which attempted to exploit this effect and allow the classification of individual airborne particles at high throughput rates was developed by Ludlow (1982). This instrument employed a spherical scattering chamber, the centre of which was coincident with the intersection of a laser beam and an orthogonal airborne

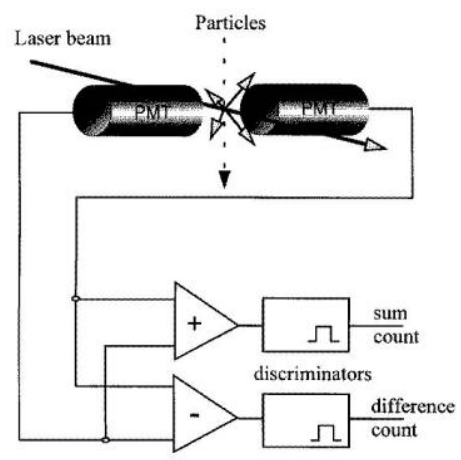

Figure 2. A schematic diagram of the instrument which Allen and Husar used to measure asymmetrical scattering from individual airborne particles. A particle which scatters symmetrically to the two detectors gives a pulse output at the 'sum count' but not at the 'difference count'; an asymmetrical scatterer gives a pulse at both outputs. The proportion of asymmetrical scatterers can thus be estimated. (Adapted from Allen and Husar (1976).) 

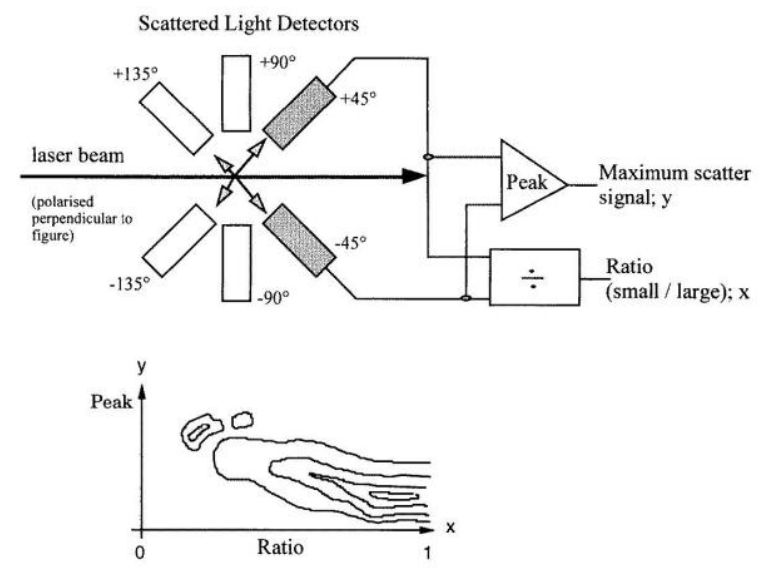

Figure 3. A schematic diagram of the instrument which Diehl et al used to distinguish fibrous from non-fibrous particles in an aqueous environment. It measured light scattered by sub- $2 \mu \mathrm{m}$ sized particles to pairs of detectors at scattering angles $\theta$ of $\pm 45^{\circ}$ , $\pm 90^{\circ},+45^{\circ}$ and $-135^{\circ}$, or $+45^{\circ}$ and $+135^{\circ}$. The plot illustrates the peak scattering signal versus the ratio of the signals as a contour map (particle frequency). This allowed detection of asbestiform fibres in filtration plant samples down to $5 \times 10^{4}$ fibres per litre. (Adapted from Diehl et al (1979).)

particle flow. The chamber incorporated an arrangement of three photomultiplier detectors arranged around the beam axis at 120 azimuthal intervals and at a scattering angle $\theta$ of $60^{\circ}$ to the beam axis. The beam itself was circularly polarized. The detectors received light scattered by the individual particles passing through the beam and, by comparison of the detector output signals, the instrument was proved capable of differentiating between spherical airborne droplets (for which the signals were equal) and other non-spherical particles, within the particle size range approximately 5-30 $\mu \mathrm{m}$.

This approach of using triple detectors arranged symmetrically about the axis of the illuminating beam of

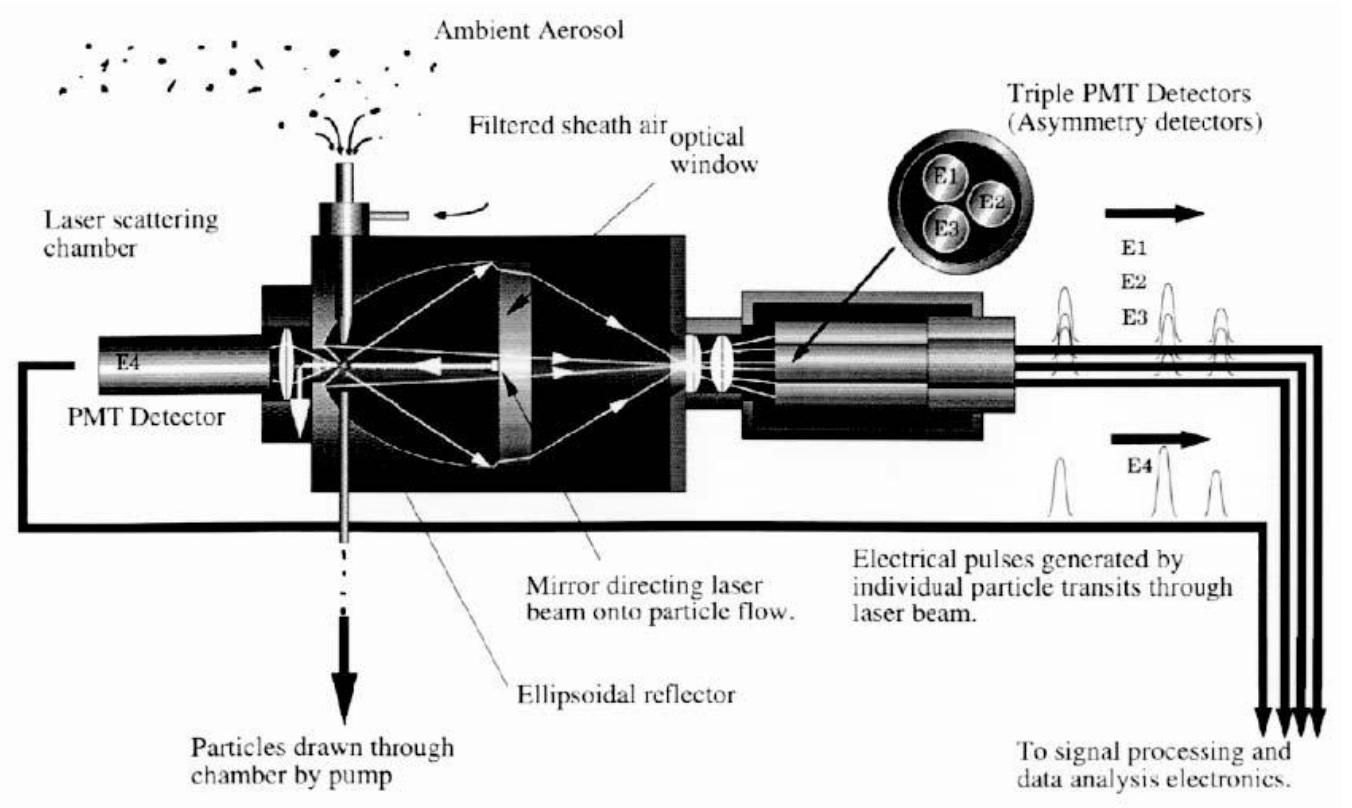

Figure 4. A schematic diagram of the instrument developed by Kaye et al (1996) which incorporated an arrangement of three miniature photomultipliers to allow measurement of variations in azimuthal scattering from individual airborne particles. The output of detector E4 which measured the forwards scattering was used in estimation of the particle size. The beam from a $670 \mathrm{~nm}$ diode laser (not shown) was directed onto the particle flow by a small 45 mirror supported by an optical window as shown.

light was further developed in the late 1980s (Kaye et al 1990, 1991) by the incorporation of an ellipsoidal reflector system which surrounded the scattering particle and allowed a far greater proportion of the spatially scattered light to be collected. This arrangement, shown schematically in figure 4, utilized three miniature photomultiplier detectors arranged symmetrically about the laser beam axis to measure azimuthal scattering variations. Each detector subtended a large solid angle centred on the scattering particle, enhancing instrument sensitivity through improved signal-to-noise ratios and allowing spheroidal particles having Feret ratios (length-to-width ratios measured from the particle silhouettes by conventional optical 
microscopy) as low as $1.08: 1$ to be discriminated from perfect spheres by inter-comparison of the three detector outputs. Although scattered light was collected throughout a range of polar scattering angle $\theta$ from approximately $28^{\prime}$ to $141^{\prime}$, namely the limits of the ellipsoidal reflector, this was simply integrated and no attempt was made to measure intensity variations over the range of $\theta$ values.

The instrument shown in figure 4 additionally incorporated a focused aerodynamic sample delivery system in which elongated particles tended to align axially with the sample airflow, thus scattering light preferentially in the horizontal plane. For such elongated particles, detector E2 (which lay in the horizontal plane) therefore gave consistently higher output signals than did the other two detectors and, from the ratios of these output signals, an assessment of the aspect ratio (length:width) of the particle could be made.

Even partial control of the orientation of non-spherical particles within the measurement space is of critical importance in spatial scattering measurements of this type and can significantly improve the efficiency of particle discrimination on the basis of shape. Without such control, particles can adopt random orientations with the result that even particles of identical shape may produce substantially differing scattering patterns, making the task of efficient shape classification virtually impossible. The facility of being able to orientate elongated particles preferentially in this way has therefore been implemented further in a variety of subsequent instruments, including a commercial variant of the system shown in figure 4, the Aerosol Shape Analyser (Bristol Industrial and Research Associates Ltd, Portishead, UK), which allows airborne particles in the sub-10 $\mu \mathrm{m}$ size range to be classified into size and shape (scattering asymmetry) classes in real-time at rates of up to about 10000 particles per second.

Although the instruments described above were able not only to differentiate between spherical and non-spherical particles but also to provide some crude indication of the particle shape (or, more correctly, scattering asymmetry), the full potential of spatial intensity scattering analysis for non-spherical particle characterization could only be realized by the detailed analysis both of azimuthal and of polar scattered intensity variations.

\subsection{Combined polar and azimuthal scattering studies}

Wyatt $e$ t $a$ l (1988) introduced the DAWN-A laser-scattering instrument. This comprised a spherical metal chamber, shown schematically in figure 5 , the centre of which was coincident with the intersection of a laser beam and an orthogonal airborne particle flow. The chamber was pierced by 72 small and two large detector apertures. The small apertures were arranged on four azimuthal planes

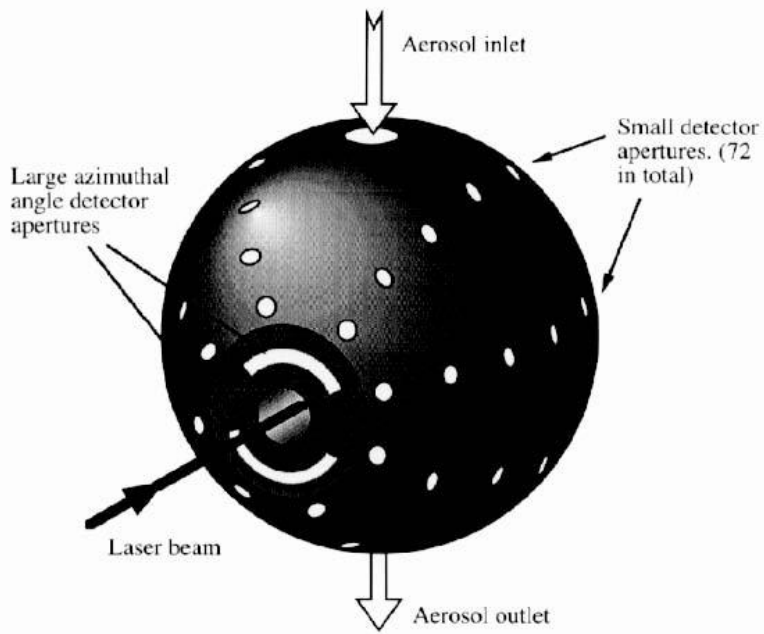

Figure 5. A simplified schematic diagram of the laser-scattering chamber of the DAWN-A instrument developed by Wyatt (1988) for polar and azimuthal scattering investigations. The apertures could be fitted with optical fibre detectors and polarization analysers or could be plugged to seal the chamber from external air. (Adapted from Wyatt et al (1988).)

(18 apertures on each), set at azimuthal intervals of $45^{\circ}$. Each small aperture could be plugged to seal the chamber from external air or could contain an optical fibre probe to convey scattered light to a discrete photomultiplier detector. Each of the two large detector apertures subtended a large azimuthal angle centred on scattering angles $\theta$ of $25^{\circ}$ and $155^{\circ}$ respectively. The instrument thus provided a flexible platform for investigations both of polar and of azimuthal scattering and has been used by many researchers. Sachweh et al (1995), for example, used the DAWN-A instrument to investigate sub-micrometre spherical and non-spherical aerosol particle scattering over a range of polar and azimuthal scattering angles. By using this approach, they were able to discriminate individual polystyrene latex spheres from similarly sized quartz and sodium chloride particles to better than $95 \%$ accuracy.

In order to facilitate fundamental studies of the spatial light-scattering behaviour of individual spherical and non-spherical airborne particles, an instrument similar to that shown in figure 4 but in which the triple-detector arrangement was replaced 
by a high-resolution charge-coupled-device (CCD) intensifier camera to allow the recording of detailed scattering profiles from individual particles has been constructed (Hirst 1996). The light falling on the camera was therefore a two-dimensional transform of the three-dimensional spatial intensity distribution falling onto the ellipsoidal reflector and corresponded to approximately $83 \%$ of the total sphere of scattering about the particle.

Figure 6 shows spatial scattering profile images from a variety of particle types recorded using this CCD-based instrument: (a) and (b) were recorded from an aerosol of $12 \mu \mathrm{m}$ long micromachined silica fibres; (c) was recorded from a water-droplet aerosol and corresponds to a droplet of $4 \mu \mathrm{m}$ diameter; (d) was recorded from a sodium chloride

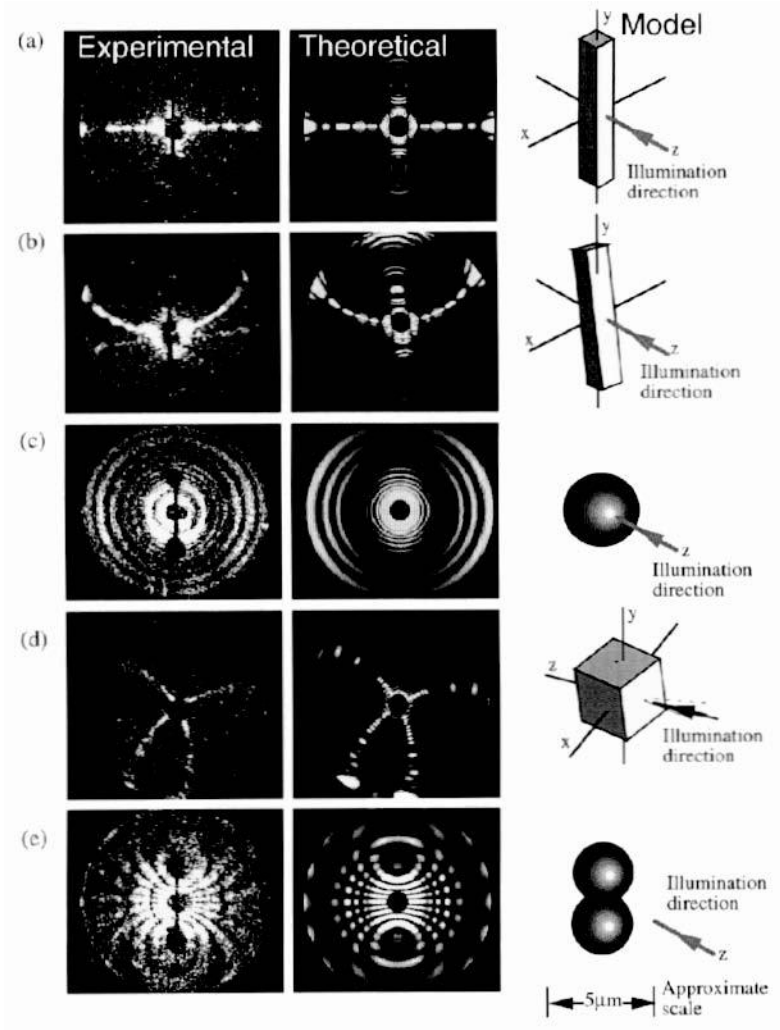

Figure 6. The left-hand column shows a variety of experimental scattering profiles recorded from individual airborne particles using a scattering chamber similar to that shown in figure 4 but equipped with an CCD intensifier camera in place of the three photomultiplier tubes. Each image therefore represents approximately $83 \%$ of the total sphere of scattering around the particle. The middle column shows the best-fit theoretical plots based on the models shown on the right-hand side.

aerosol and corresponds to a cubic crystal of about $5 \mu \mathrm{m}$ sidelength; and (e) was recorded from a $3 \mu \mathrm{m}$ polystyrene latex sphere aerosol in which approximately $8 \%$ of the observed particles were doublets of the type shown. The innermost dark circle in the images results from the aperture in the rear of the ellipsoidal reflector and its circumference corresponds to a scattering angle of 28 to the beam axis; the outer circumference of the images corresponds to scattering at $141^{\circ}$ to the beam axis. Each white dot in the images corresponded to a single photon-scattering event. The dark vertically oriented 'dumb-bell' shadow was an artefact of the instrument, the circular regions being caused by the ends of the airflow inlet and outlet tubes and as such their centres corresponded to scattering at 90 directly above and below the particle respectively.

Figure 6 also gives corresponding best-fit theoretical scattering profiles rendered as grey-scale images and the corresponding physical particle geometries used in the generation of these theoretical models. The theoretical profiles were produced for spherical particles by computing the full angular intensity functions from Mie scattering theory (Bohren and Huffman 1983) and modifying these to take into account the geometrical distortion produced by the optical configuration of the scattering chamber. The non-spherical particles were modelled using an approach based on Rayleigh-Gans scattering in which the particle was modelled as a three-dimensional array of independent isotropic scattering points. This procedure has been described in more detail by Hirst et al (1994).

Images of the type shown in figure 6 have proved valuable in fundamental studies of particle spatial scattering and have shown that, for regular particle geometries at least, theoretical models provide a reasonable vehicle for pattern interpretation. The examples also illustrate not only the wide variety of scattering profiles observable from different particle shapes but also, as illustrated in figures 6(a) and (b), recorded from essentially identical particles, the pronounced effect which particle orientation has on the scattering profiles of non-spherical particles. 


\section{A practical application: asbestos monitoring}

Asbestos has been used widely throughout the industrialized world for thermal insulation, fire protection, material strengthening and so on. Even though its use is now banned, airborne fibres of asbestos can be generated whenever buildings or plant containing the material are refurbished or demolished. These airborne asbestos fibres pose a significant health risk if inhaled. The mortality rate from asbestos-related diseases is still rising in the UK (Peto et al 1995) and the USA National Institute for Occupational Safety and Health stated in 1990 that there is "no evidence for a threshold or "safe' level of asbestos exposure" (NIOSH 1990). The need for in situ detection of airborne asbestos fibres has therefore attracted considerable attention.

Conventional monitoring techniques are based upon filter sampling of the contaminated environment followed by optical or electron microscopy analysis of particles entrapped on the filter. Fibrous particles are counted manually or by computer image processing in accordance with strict counting rules governing the sizes and aspect ratios of fibres to be included; typically lengths in the range 5-20 $\mu \mathrm{m}$ and aspect ratios of at least $3: 1$. This can be laborious and time consuming and provides results long after inadvertent exposure of personnel in or around the sampling site may have occurred. Spatial light scattering was recognized at an early stage as a method which could offer real-time discrimination of airborne fibres, especially because of the highly characteristic scattering which such particles produce (as indicated in figure 6). An elegant instrument which exploited this characteristic scattering in airborne fibre measurement is the FAM-7400 Fibrous Aerosol Monitor (Mie Inc, Bedford, Massachusetts, USA) developed originally by Lilienfeld (Lilienfeld et $a$ 1979, Lilienfeld 1987). This instrument, shown schematically in figure 7 , draws air containing the airborne particles through a laser-scattering chamber which is enveloped by a quadruple electrode arrangement. By applying a time-varying signal to the electrodes, the electric field within the scattering chamber causes conducting fibres present in the air to oscillate. (Asbestos fibres generally fall into this category because of their high water adsorption.) The consequent cyclical variation in light scattered by the fibres

(a)

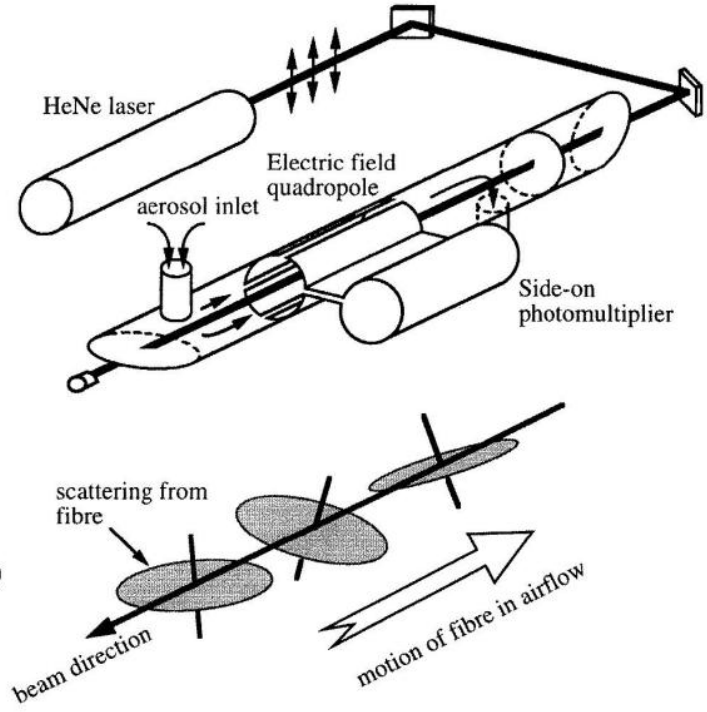

Figure 7. (a) A schematic diagram of the airborne fibre-sensing system developed by Lilienfeld. As a fibre moves through the scattering volume, it oscillates under the influence of the applied field as shown in (b) and the scattered light (predominantly in the plane orthogonal to the fibre axis) transiently reaches the PMT detector, causing a modulation of the detector output. (Adapted from Lilienfeld (1987).)

to a single slit-shaped light detector at the side of the chamber is used to assess the concentration of fibres in the air.

An alternative approach has been used by the author and co-workers (Hirst et al 1995, Kaye et al 1997), in which the spatial scattering profile from individual particles is analysed by an artificial neural network technique in order to classify the particles according to characteristic features of the profiles. The instrument, shown schematically in figure 8 , records the forwards spatially scattered light from individual particles traversing the beam from a diode laser using a dedicated photodiode array chip. (The detector chip, shown also in figure 8 , is configured as two 16segment concentric rings surrounding a single continuous annular ring. For further details relating to this design and to the instrument's performance see Kaye et al (1997).) The scattering profiles recorded by this instrument cover a much smaller scattering angle range than do those shown in figure 6 , the polar scattering being restricted to angles less than approximately $30^{\circ}$. This simplifies the instrument's geometry and also results in profiles less affected by slight misalignment of elongated or fibrous particles (Kaye et al 1997).

Figure 9 illustrates some scattering profiles recorded from individual airborne particles using this instrument. (Note that these illustrative examples were produced by temporarily replacing the photodiode chip by a high-resolution intensified CCD camera.) The top row in figure 9 shows the spatial scattering profiles from a variety of typical 'background' particles; the middle row shows scattering profiles recorded from individual particles of crocidolite (blue) asbestos, one of the most carcinogenic 


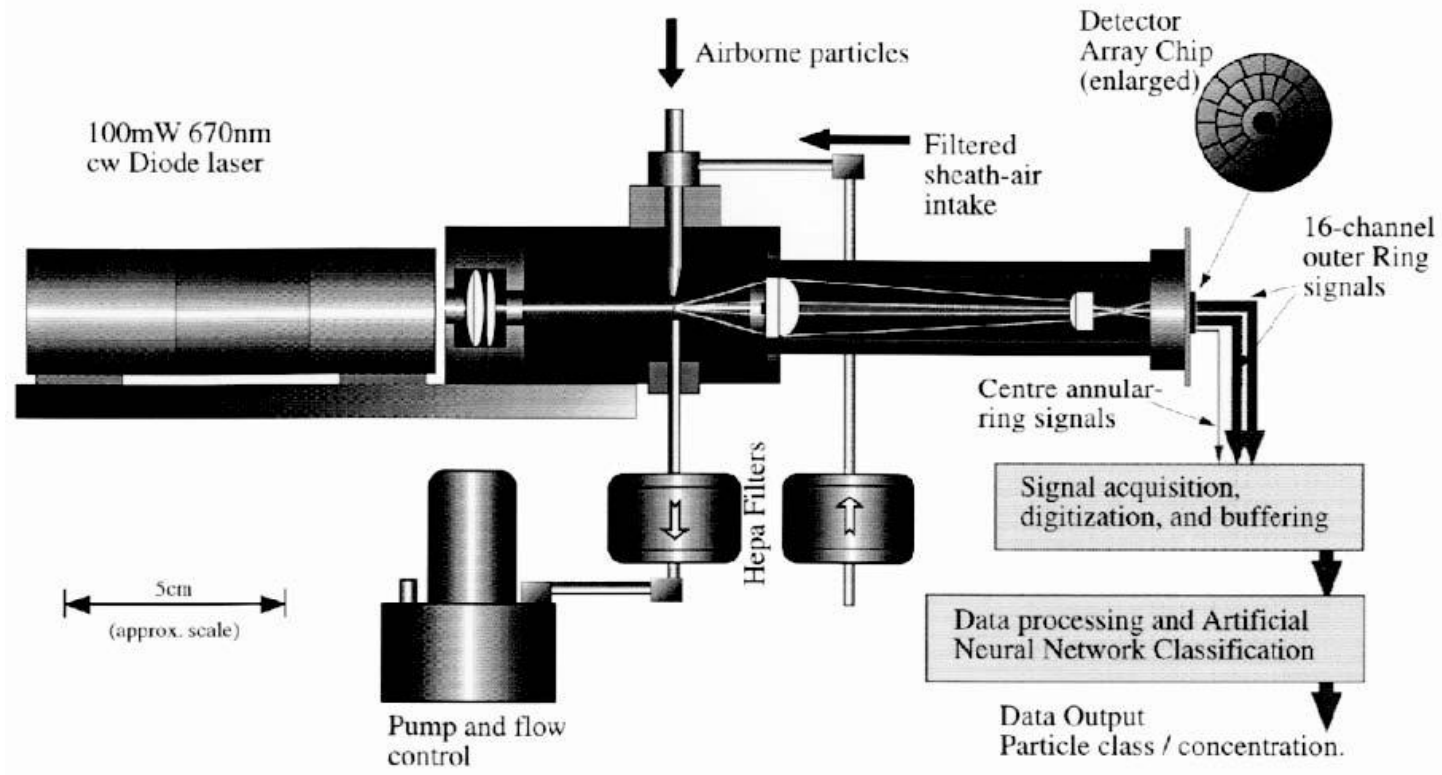

Figure 8. A schematic diagram of the laser-scattering instrument used for the real-time detection of airborne asbestos fibres. The forwards scattering profile is recorded using a dedicated detector-array chip as shown and these data are processed using a neural network classifier previously trained to recognize characteristic features in the spatial scattering profiles of asbestos.

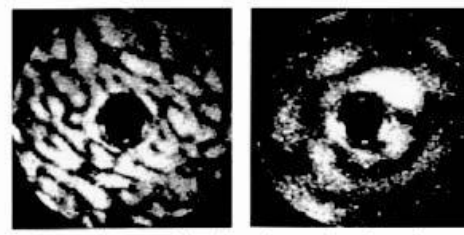

Scattering profiles from irregular shaped background particles.
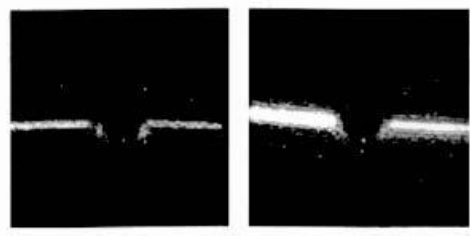

Scattering profiles from individual crocidolite asbestos fibres

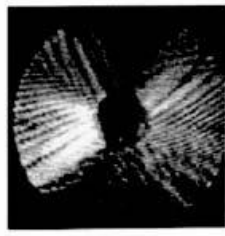

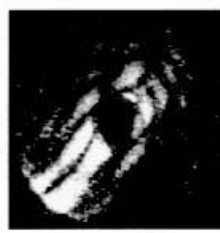

Flake particle
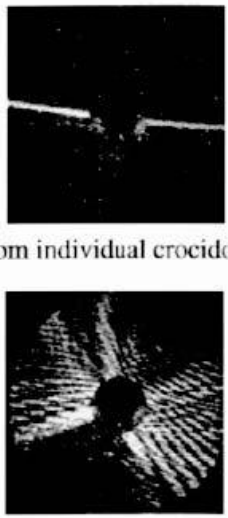

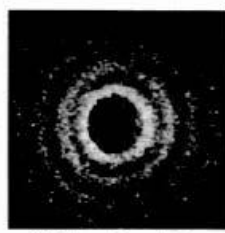

Water droplet ( $\sim 9 \mu \mathrm{m}$ dia).
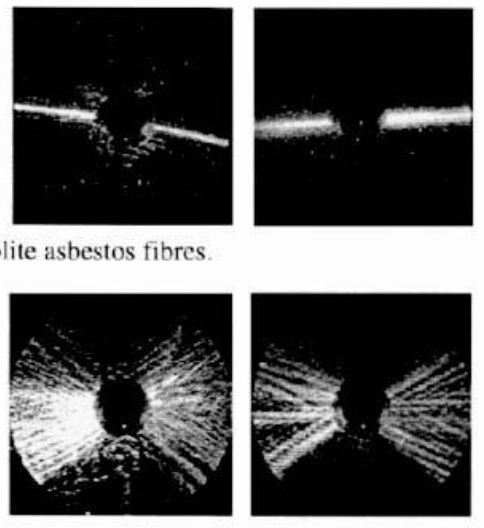

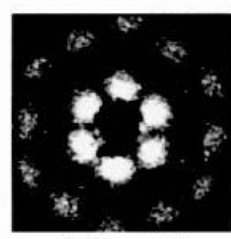

Salt crystal (corner-on)

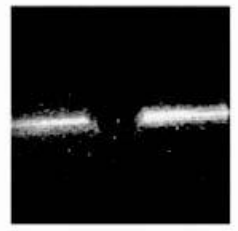

Scattering profiles from individual chrysotile asbestos fibres.

Figure 9. Scattering profiles from individual airborne particles recorded by the instrument shown in figure 8 but using a CCD intensifier camera as the detector rather than the photodiode-array chip. The upper row illustrates the wide variety of profiles derived from typical 'background' particles; the centre row shows profiles from individual crocidolite asbestos fibres; and the lower row shows typical 'bow-tie' profiles characteristic of the curved fibres of chrysotile asbestos.

forms; the bottom row shows scattering from fibres of two materials exhibit significant characteristic differences chrysotile (white) asbestos, which is less carcinogenic but in their scattering profiles. In the chrysotile cases, the 'bowfar more abundant, being found in about $95 \%$ of all asbestos tie' shaped scattering is as a result of the natural curvature installations. Both types of fibre result in predominantly exhibited by chrysotile fibres; in the crocidolite cases, the horizontal scattering (caused by the preferential vertical fine scattering structure reflects the straight, needle-like, alignment of the fibres in the sample airflow), but the fibre geometries common for this material. 


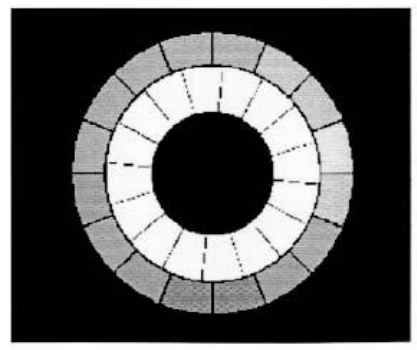

Water droplet

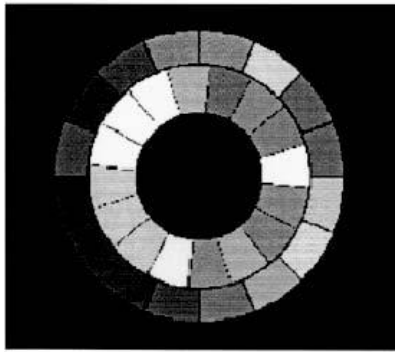

Irregular 'background' particle.

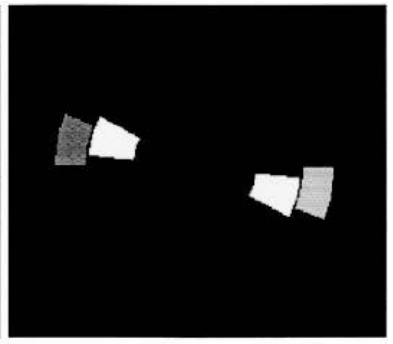

Crocidolite asbestos

fibre.

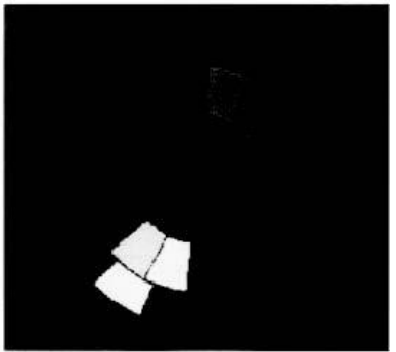

Flake-like particle

Figure 10. Grey-scale representations of the spatial scattered light distributions from individual airbome particles recorded using the detector chip shown in figure

8. Data of this type are fed to the neural network processor which classifies the particles according to characteristic scattering-pattern features.

Figure 10 shows actual individual particle scattering data, rendered as grey-scale images, recorded by the instrument (shown in figure 8) when fitted with its custom photodiode array detector. Data of this form are analysed using a radial basis function neural network (Schalkoff 1992) which classifies the scattering patterns into predefined classes. These classes are defined by 'training' the instrument using known aerosols of crocidolite and chrysotile asbestos, such that the neural network is subsequently capable of recognizing features within the scattering profiles which are characteristic of each of these materials. Individual fibres in an environment under test may thus be detected against a background of other particulates, thus providing a real-time monitoring capability.

It is worthy of note that the use of a 'trained' neural network classifier in this way has the advantage that it does not attempt to relate the scattering profile to a specific particle geometry (which would be the inverse problem), a task which is computationally intensive even for comparatively simple particle geometries. Instead, the scattering profiles are treated as abstract data which are classified according to stored weighting functions previously derived from the training data. In theory, the network may be trained to recognize any type of particle which can be generated as a test aerosol, thus allowing the acquisition of training data. However, the accuracy of its subsequent classification process will depend ultimately on the extent to which the inevitable spread of scattering profiles from the particles of interest overlaps with that resulting from the diverse background particle population.

\section{Summary}

The analysis of the spatial intensity distribution of light scattered by individual particles can provide a powerful means of discriminating or classifying the particles. This review has briefly touched on some of the areas in which this approach, offering rapid, non-destructive analysis, can be of value for monitoring and characterizing air or liquid-borne particulates. As in many other areas of analytical science, a limitation to wider exploitation of the technology of spatial scattering analysis has been the considerable problems associated with the volume of data produced, especially when real-time monitoring or process control is required, and the commensurate need for powerful (and possibly costly) computer-processing resources. The continuing decline in the cost of dedicated computers, coupled with advances in compact diode laser and photodetector technologies, is beginning to overcome this limitation and opportunities for exploitation in new areas of particulate characterization are continually arising. Furthermore, interest in the development of instruments which employ other physical and/or chemical analytical techniques (such as, for example, Raman scattering or fluorescence) in combination with spatial 
scattering analysis is increasing. Such instruments may offer significantly enhanced particle-discrimination capabilities and could find application in a wide range of environmental and pollution-monitoring fields.

\section{References}

Al-ChalabiSAM, Jones A R, Savaloni H and Wood R 1990 Meas. Sci. Technol. 129-35

Allen J and Husar R B 1976 Proc. Int. Conf. on Colloid and Interface Science. vol 2, ed M Kerker (New York: Academic) pp 141-53 Bartholdi M, Salzman G C, Hiebert R D and Kerker M 1980 Appl. Opt. 19 1573-81

Bohren C F and Huffman D R 1983 Absorption and Scattering of Light by Small Particles (New York: Wiley)

Bottlinger M and Umhauer H 1987 Proc. Int. Congress on Optical Particle Sizing (New York: Plenum) pp 363-9

Diehl S R, Smith D T and Sydor M 1979 Appl. Opt. $181653-8$

Gebhart J 1976 Fine Particles edBYHLiu (New York: Academic) pp 793-815

Gebhart J and Anselm A 1987 Proc. Int. Congress on Optical Particle Sizing (New York: Plenum) pp 393-409

HeffelsMGC, Heitzmann D, Hirleman E D and Scarlett B 1994 Part. Part. Syst. Charact. 11 194-9

Hirst E and Kaye P H 1996 J. Geophys. Res. (Atmospheres) 101 D 19 231-5

Hirst E, Kaye P H and Guppy J 1994 Appl. Opt. $33307180-6$

Hirst E, Kaye P H and Hoskins J A 1995 Ann. Occupational Hygiene 39 623-32

Kaye P H, Eyles N A and Clark J M 1990 Proc. 2nd Int. Congress on Optical Particle Sizing (Phoenix, AZ: University of Arizona) pp 501-10

Kaye P H, Eyles N A, Ludlow I K and Clark J M 1991 Atmospheric Environment A 25 645-54

Kaye P H, Hirst E and Wang-Thomas Z 1997 Appl. Opt. 36 6149-56

Killinger R T and Zerull R H 1987 Proc. Int. Congress on Optical Particle Sizing (New York: Plenum) pp 419-29

Latimer P, Pyle B, Moore C and Brunsting A 1978 Appl. Opt. 17 3152-9

Lilienfeld P 1987 J. Aerosol Sci. 18 389-95

Lilienfeld P, Elterman P and Baron P 1979 American Industrial Hygiene Assoc. J. 40 270-82

Ludlow I K 1982 Particle analysis by light scattering UK Ministry of Defence contract report ER1A/9/4/2161/CDE

Ludlow I K and Kaye P H 1979 J Coll. Interface Sci. 69 571-89

Neeße T, D“uck J and Thaufelder T 1995 Part. Part. Syst. Charact. 13 295-300

NIOSH 1990 29CFR parts 1910 and 1926

Peto J, Hodgson J T, Matthews F E and Jones J R 1995 The Lancet 345 535-9

Rarity J 1987 Proc. Int. Congress on Optical particle Sizing (New York: Plenum) pp 217-27

Rochon P, Racey T J and Zeller M 1988 Appl. Opt. 27 3295-8

Sachweh B A, Dick W D and McMurry P H 1995 Aerosol Sci. Technology 23 373-91

Schalkoff R 1992 Pattern Recognition, Statistical, Structural, and Neural Approaches (New York: Wiley) pp 204-63

Spinrad R W and Brown J 1993 Appl. Opt. 32 6151-8

Ulanowski Z and Ludlow I K 1993 Current Microbiol. 26 31-5

Umhauer H and Bottlinger M 1991 Appl. Opt. 30 4980-6

Wyatt P J 1968 Appl. Opt. 7101972 J. Colloid Interface Sci. 39 125-35

Wyatt P J, Schehrer K L, Phillips S D, Jackson C, Chang Y-J, Parker R G, Phillips D T and Bottiger J R 1988 Appl. Opt. $27217-21$

Zerull R H, Killinger R and Weiss-Wrana K 1986 Preprints, Part 1, 1st World Congress on Particle Technology, PARTEC (N"urnberg) pp 141-4 